\title{
Topological photonic crystals with zero Berry curvature
}

\author{
Feng Liu, ${ }^{1}$ Hai-Yao Deng, ${ }^{2}$ and Katsunori Wakabayashi ${ }^{1,3}$ \\ ${ }^{1}$ Department of Nanotechnology for Sustainable Energy, School of Science and Technology, Kwansei Gakuin University, \\ Gakuen 2-1, Sanda 669-1337, Japan \\ ${ }^{2}$ School of Physics, University of Exeter, Stocker Road EX4 4QL Exeter, United Kingdom \\ ${ }^{3}$ National Institute for Materials Science, Namiki 1-1, Tsukuba 305-0044, Japan
}

(Received 26 November 2017; published 31 January 2018)

\begin{abstract}
Topological photonic crystals are designed based on the concept of Zak's phase rather than the topological invariants such as the Chern number and spin Chern number, which rely on the existence of a nonvanishing Berry curvature. Our photonic crystals (PCs) are made of pure dielectrics and sit on a square lattice obeying the $C_{4 v}$ point-group symmetry. Two varieties of PCs are considered: one closely resembles the electronic two-dimensional $\mathrm{Su}-$ Schrieffer-Heeger model, and the other continues as an extension of this analogy. In both cases, the topological transitions are induced by adjusting the lattice constants. Topological edge modes (TEMs) are shown to exist within the nontrivial photonic band gaps on the termination of those PCs. The high efficiency of these TEMs transferring electromagnetic energy against several types of disorders has been demonstrated using the finite-element method.
\end{abstract}

DOI: 10.1103/PhysRevB.97.035442

\section{INTRODUCTION}

The topological classification of crystals regarding their energy band structures has renewed our understanding of solid-state materials [1-5]. One unique realization in this context is the occurrence of states propagating around the interfaces between topologically nonequivalent systems. These topologically protected edge modes (TEMs) are impervious to weak disorders and thus have been pursued vigorously for the realization of ultralow-power electronic devices as well as fundamental blocks of quantum computation. Examples of topologically nontrivial systems hosting TEMs include quantum Hall [6] and quantum spin Hall systems [7-13] besides the more recently realized ones utilizing pseudospins such as degenerate atomic orbitals [14-16] and chiralities [17-19]. Most topologically nontrivial systems require a nonvanishing Berry curvature: the "magnetic field" defined in momentum space [20-22].

In a recent paper, however, two of us have shown that even with zero Berry curvature, topologically nontrivial electronic band structures can emerge in a simple square lattice without (pseudo)spins. [23] The topology is characterized by an integration of the Berry connection, the "magnetic vector potential" whose curl gives the Berry curvature [22], over the momentum space resulting in a nontrivial Zak's phase. This intriguing scenario reminds us of the Aharonov-Bohm effect in a topological sense and can be extended to other particles like photons. In fact, almost every electronic topological material has already found its photonic counterpart, such as Chern insulators [24-31], spin Chern insulators [32-38], and Weyl semimetals $[39,40]$.

In this work, we demonstrate the nontrivial topological system in the absence of Berry curvatures using photonic crystals made of dielectrics (DPCs). In DPCs, photonic band structures for macroscopic electromagnetic (EM) waves naturally replace electronic band structures in solids, and photonic TEMs are hence expected. In comparison with electronic materials, DPCs are relatively easier to fabricate and thus provide an attractive option for experimental studies. Several experimentally feasible designs of DPCs with zero Berry curvature but robust TEMs are proposed, which not only corroborates the discovery made in Ref. [23] but also has direct experimental relevance.

Our designed DPC consists of a single type of dielectric like silicon arranged in a two-dimensional (2D) periodic array endowed with $C_{4 v}$ point-group symmetry (PGS). Topologically nontrivial photonic band gaps are shown to exist in this crystal and are protected by the nonvanishing vectored Zak's phase. We numerically solve Maxwell's equations using the finite-element method (FEM) and demonstrate that TEMs emerge in the photonic band gaps when the DPC is terminated by the topologically trivial ones. These TEMs are shown to be robust against various kinds of disorders, such as lattice imperfections, broken units, metallic obstructions, and orthogonal corners. Our results confirm the existence of a nontrivial topological phase with zero Berry curvature in photonic crystals and offer a means of building efficient photon transport channels.

This paper is organized as follows. In Sec. II, we briefly introduce the vectored Zak's phase through a 2D Su-SchriefferHeeger (SSH) model. In Sec. III, we derive a tight-binding formalism for EM waves in DPCs and propose a DPC that resembles the 2D SSH model. In Sec. IV, we continue designing another DPC with the so-called Gonome structure as the extension of the 2D SSH model and demonstrate its nontrivial topological properties. We discuss the results and summarize the paper in Sec. V. A few appendixes are provided as a supplement to the discussion in the main text.

\section{TOPOLOGY WITH ZERO BERRY CURVATURE: 2D SSH MODEL}

The scenario of topologically nontrivial structures with zero Berry curvature can be readily elucidated with a simple tightbinding model on a square lattice as sketched in Fig. 1(a) [23]. 


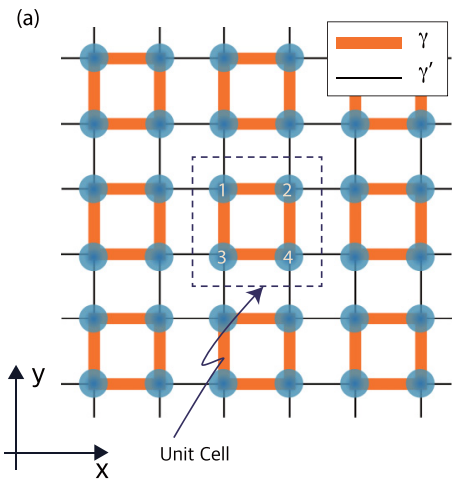

(b)

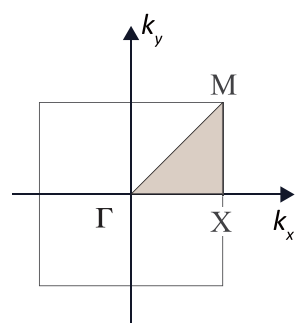

(c)

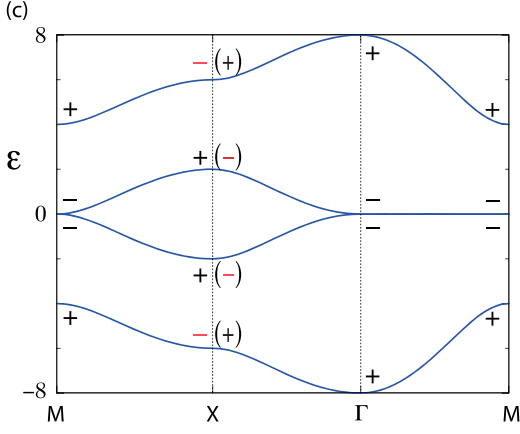

(d)

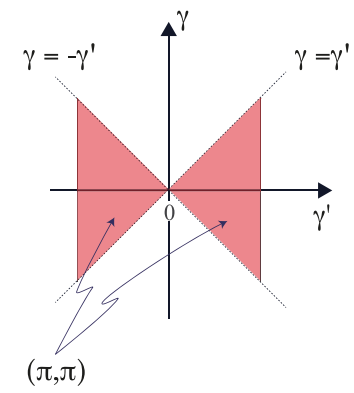

FIG. 1. (a) Schematic lattice structure of the 2D SSH model described by Eq. (1). The model is specified by the intracellular hopping $\gamma$ and the intercellular hopping $\gamma^{\prime}$. The topological properties are characterized by the vectored Zak's phase in this model. (b) Corresponding first BZ. (c) Band structures of topologically nontrivial phases, i.e., $\gamma=1.0$ and $\gamma^{\prime}=3.0$. The topologically trivial energy band structures are identical to the topologically nontrivial ones for swapped $\gamma$ and $\gamma^{\prime}$, except for the parities at point $X$. The opposite parities at $X(Y)$ and $\Gamma$ give rise to the vectored Zak phase $(\pi, \pi)$ in the nontrivial case and $(0,0)$ in the trivial case. The signs in the parentheses indicate the parity for the trivial case, i.e., $|\gamma|^{\prime} /|\gamma|<1$. (d) Topological phase diagram in terms of the vectored Zak's phase. Nontrivial phases with a Zak's phase of $(\pi, \pi)$ appear for $\left|\gamma^{\prime}\right| /|\gamma|>1$.

This model represents a 2D generalization of the Su-SchriefferHeeger (SSH) model and is specified with two parameters, i.e., the intracellular hopping $\gamma$ and the intercellular hopping $\gamma^{\prime}$. In the $x$ and $y$ directions they are indicated by thick red and thin black lines in Fig. 1(a), respectively. The energy band structure of this model is obtained by solving the following Hamiltonian $\mathcal{H}(\mathbf{k})$ in $\mathbf{k}$ space:

$$
\left(\begin{array}{cccc}
0 & \gamma+\gamma^{\prime} \exp \left(i k_{x}\right) & \gamma+\gamma^{\prime} \exp \left(-i k_{y}\right) & 0 \\
\gamma+\gamma^{\prime} \exp \left(-i k_{x}\right) & 0 & 0 & \gamma+\gamma^{\prime} \exp \left(-i k_{y}\right) \\
\gamma+\gamma^{\prime} \exp \left(i k_{y}\right) & 0 & 0 & \gamma+\gamma^{\prime} \exp \left(i k_{x}\right) \\
0 & \gamma+\gamma^{\prime} \exp \left(i k_{y}\right) & \gamma+\gamma^{\prime} \exp \left(-i k_{x}\right) & 0
\end{array}\right) \Psi=\varepsilon \Psi,
$$

where $\Psi=\left(\phi_{1}, \phi_{2}, \phi_{3}, \phi_{4}\right)$ are the bases of the atomic sites in a unit cell numbered from 1 to 4 , as shown in Fig. 1(a), and $k_{x}$ and $k_{y}$ are the wave numbers along the $x$ and $y$ directions in the first Brillouin zone (BZ), as shown in Fig. 1(b).

One can show that due to inversion and time-reversal symmetries the Berry curvature vanishes everywhere in the first BZ without band degeneracy, and its average over the first BZ vanishes even where energy bands are degenerate $[22,23]$. The topologically nontrivial transition from the case of $|\gamma|>\left|\gamma^{\prime}\right|$ to that of $\left|\gamma^{\prime}\right|>|\gamma|$ can be revealed in the vectored Zak's phase $\mathbf{P}=\iint d k_{1} d k_{2} \operatorname{Tr}(\mathbf{A})$, defined as an integration of the non-Abelian Berry connection $\mathbf{A}_{m n}=i\left\langle u_{m}\left|\partial_{\mathbf{k}}\right| u_{n}\right\rangle$ over the first BZ, with $\left|u_{n}\right\rangle$ being the Bloch function for the $n$th energy band and $m, n$ running over occupied bands. Because of the $C_{4 v}$ symmetry, this quantity can easily be evaluated by the following formula [41]:

$$
P_{i}=\pi\left(\sum_{n} q_{i}^{n} \bmod 2\right), \quad(-1)^{q_{i}^{n}}=\frac{\eta_{n}\left(X_{i}\right)}{\eta_{n}(\Gamma)},
$$

where $i$ stands for direction $x$ or $y, X_{i}$ denotes the $X$ or $Y$ point of the first BZ, $\eta_{n}(\mathbf{k})$ is the parity under $\pi$ rotation of the wave function for the $n$th band at symmetry point $\mathbf{k}$, and the summation runs over all occupied bands.

An example of the resulting band structure is displayed in Fig. 1(c). According to the character table of the group $C_{4 v}$, the four energy bands from top to bottom at point $\Gamma$ are an $A_{1}$ mode, a pair of $E$ modes, and a $\mathrm{B}_{1}$ mode, respectively. As with the one-dimensional (1D) SSH model, the band dispersions remain unchanged if $\gamma$ and $\gamma^{\prime}$ are swapped. However, the wave functions are qualitatively different: they possess opposite parities at $X$. As a result, the vectored Zak's phase $\mathbf{P}=$ $(\pi, \pi)$ for $\left|\gamma^{\prime}\right| \gamma \mid>1$ but $(0,0)$ otherwise, as indicated by the topological phase diagram shown in Fig. 1(d) as far as the lowest energy band is occupied. When the periodic lattice is terminated, TEMs emerge for the topologically nontrivial phase, and their properties have been extensively studied in our previous work [23]. A similar model defined on a Kekulé lattice including a small next-nearest-neighbor hopping has also been studied, and a similar scenario has been observed [42,43].

Here let us mention the physical meaning and bulk-edge correspondence of Zak's phase. The presence of Zak's phase is related to charge polarization $\left(\sigma_{i}\right.$ with $\left.i=x, y\right)$ through $\sigma_{i}=P_{i} / 2 \pi$ [41,44-46]. Since Zak's phase has only two possible values, 0 or $\pi$, under the constraint of inversion symmetry, the corresponding charge polarization is quantized as 0 and $1 / 2$ (charge polarization is well defined up to 1 in units of lattice constant $a$ ). For Zak's phase $(\pi, \pi)$, the charge polarization is $(1 / 2,1 / 2)$ in both the $x$ and $y$ directions, which is a consequence of energy band inversion, i.e., the alternation of parities at inversion-invariant points of $\mathrm{BZ}$ as indicated in Eq. (2) [41,47]. Further, according to the multipole expansion of dielectrics, charge polarization $\sigma_{i}$ is related to 


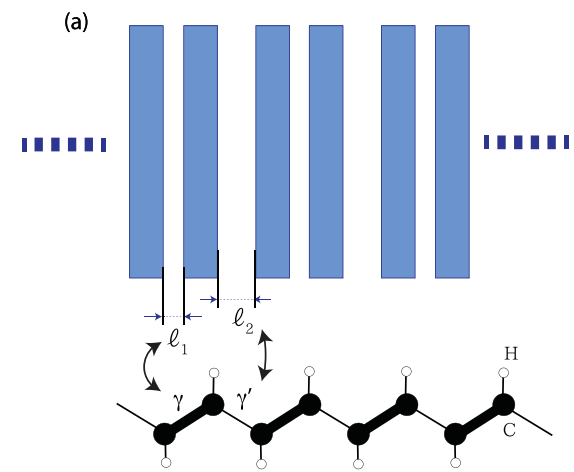

(b)

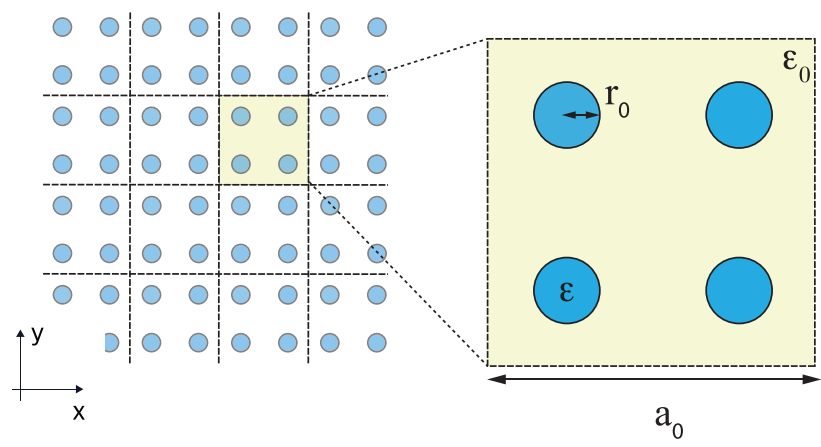

FIG. 2. (a) Schematic of the DPC mimicking the 1D SSH model. It consists of a $1 \mathrm{D}$ array of dielectric sheets with alternating spacings $l_{1}$ and $l_{2}$. (b) Schematic of the Shinome DPC mimicking the 2D SSH model. The unit cell consists of four identical dielectric cylinders of radius $r_{0}$ placed at $( \pm 0.25, \pm 0.25) r_{0}$. The lattice constant is $a_{0}$.

charge accumulation on the surface with the same value [45]. These edge states are also doubly degenerate, corresponding to even and odd parities of the inversion operation. In general, $\left|\gamma^{\prime}\right|-|\gamma|$ determines the robustness of these edge states against perturbations (see the Supplemental Material of Ref. [23]). $C_{4}$ PGS guarantees that the edge states can appear in an arbitrary direction $\left(\sigma_{i}=\mathbf{P} \cdot \mathbf{n}\right.$, with $\mathbf{n}$ being the surface direction). Inversion symmetry protects the degeneracy of these edge states. Even if both of these symmetries are broken, the edge states still exist in the $x$ or $y$ direction without degeneracy when $\left|\gamma^{\prime}\right|>|\gamma|$.

It is worth noting that in the present example the concept of spin or pseudospin does not play any essential role, which, however, has been central to a vast number of existing works. It may not be easy to induce the topological transition by tuning $\gamma / \gamma^{\prime}$ in electronic solids. Yet it may well be done in photonic crystals. In the next section, we show that the same tight-binding model, together with its topological properties, can emerge in a variety of photonic crystals.

\section{PHOTONIC 2D SSH MODEL}

DPCs modulate EM waves by dielectrics as artificial atoms and endow them with photonic band structures. In this work we are mainly concerned with transverse-magnetic EM waves in 2D DPCs, for which only the $z$ component of the electric field needs to be considered. Let us denote it by $E(\mathbf{r})$, which solves the following equation in natural units:

$$
-\partial^{2} E(\mathbf{r})=\omega^{2} \epsilon(\mathbf{r}) E(\mathbf{r}), \quad \mathbf{r}=(x, y),
$$

where $\partial^{2}=\partial_{x}^{2}+\partial_{y}^{2}, \omega$ is the frequency of the wave, and $\epsilon(\mathbf{r})$ is the dielectric function of the photonic crystal, which is real for DPCs without dissipation. Equation (3) has a unique scaling property, i.e., $(x, y) \rightarrow \alpha(x, y)$ and $\omega \rightarrow \alpha^{-1} \omega$, with $\alpha$ denoting the scaling parameter. Therefore, we can tune the optimal frequency range of DPC by properly adjusting the size and geometry of dielectric materials in DPC.

$E(\mathbf{r})$ can be expanded in terms of a complete set of Wannier functions $E_{n}(\mathbf{r}-\mathbf{R})$, i.e.,

$$
E(\mathbf{r})=\sum_{n} \sum_{\mathbf{R}} A_{n \mathbf{R}} E_{n}(\mathbf{r}-\mathbf{R}),
$$

where $\mathbf{R}$ labels the unit cells and $n=1,2, \ldots, N$, with $N$ being the number of total photonic bands. For maximally localized Wannier functions, $n$ usually differs from the band index $[48,49]$. These functions are highly localized about the corresponding unit cells and are orthonormal in terms of the following inner product:

$$
\int d^{2} \mathbf{r} E_{n}(\mathbf{r}-\mathbf{R}) \epsilon(\mathbf{r}) E_{n^{\prime}}\left(\mathbf{r}-\mathbf{R}^{\prime}\right)=\delta_{n, n^{\prime}} \delta_{\mathbf{R}, \mathbf{R}^{\prime}} .
$$

Now we substitute the expansion (4) into (3) and project to $E_{n}(\mathbf{r}-\mathbf{R})$ to obtain

$$
\begin{aligned}
\omega^{2} A_{n \mathbf{R}} & =\sum_{n^{\prime} \mathbf{R}^{\prime}} \Theta_{n \mathbf{R}, n^{\prime} \mathbf{R}^{\prime}} A_{n^{\prime} \mathbf{R}^{\prime}}, \\
\Theta_{n \mathbf{R}, n^{\prime} \mathbf{R}^{\prime}} & =-\int d^{2} \mathbf{r} E_{n}(\mathbf{r}-\mathbf{R}) \partial^{2} E_{n^{\prime}}\left(\mathbf{r}-\mathbf{R}^{\prime}\right),
\end{aligned}
$$

which is formally the same as the tight-binding model used for calculating the electronic band structure in solids. The band structure is then established by Bloch's theorem, by which one writes $A_{n \mathbf{R}}=A_{n} e^{i \mathbf{k} \mathbf{R}}$, with $\mathbf{k}$ being the wave vector in the first BZ. Equation (6) then becomes

$$
\omega^{2} A_{n}=\sum_{n^{\prime}} \Theta_{n n^{\prime}}(\mathbf{k}) A_{n^{\prime}}, \quad \Theta_{n n^{\prime}}(\mathbf{k})=\sum_{\mathbf{R}^{\prime}} e^{i \mathbf{k}\left(\mathbf{R}^{\prime}-\mathbf{R}\right)} \Theta_{n \mathbf{R}, n^{\prime} \mathbf{R}^{\prime}}
$$

where the matrix $\Theta(\mathbf{k})$ plays a role similar to $\mathcal{H}(\mathbf{k})$ in Eq. (1). This equation determines the band structure of a DPC [50].

For the purpose of illustration of Eq. (7), let us take the photonic analog of the SSH model [44], namely, an array of dielectric slabs made of the same materials with alternating spacings $l_{1}$ and $l_{2}$ [see Fig. 2(a)]. Each unit cell is composed of two slabs, which suggests that there are two independent EM modes in a unit cell. We may take these two EM modes as the basis. Within the nearest-neighbor approximation, we see that $\Theta(k)$ takes on the following form:

$$
\Theta(k)=\left(\begin{array}{cc}
\omega_{0}^{2} & \gamma+\gamma^{\prime} e^{-i k a} \\
\gamma+\gamma^{\prime} e^{i k a} & \omega_{0}^{2}
\end{array}\right),
$$

with $\omega_{0}$ being the independent mode frequency and $\gamma$ and $\gamma^{\prime}$ being the transfer integrals between slabs separated by distances $l_{1}$ and $l_{2}$, respectively. This is formally the same as the tight-binding Hamiltonian of the SSH model. 
The generalization of the above 1D example to a 2D DPC is straightforward [51]. The resulting structure is called Shi$\mathrm{No}-\mathrm{Me}$ in Japanese, as shown in Fig. 2(b). The Shinome DPC is made of four dielectric cylinders located at the four corners in a square unit cell. The tight-binding matrix $\Theta(\mathbf{k})$ of the Shinome DPC has the same form as Eq. (1), which possesses a topological transition characterized by the vectored Zak's phase rather than the Berry curvature, as discussed in Sec. II and previous work $[23,42]$. The details of the Shinome DPC are given in Appendix A.

In the next section, we design another 2D DPC as an extension of 2D SSH model. In Japanese, it is called Go-No$M e$. More than four EM energy bands are needed to understand the topological features, and the corresponding minimal $\Theta(\mathbf{k})$ has a much bigger dimension.

\section{EXTENSION OF THE PHOTONIC 2D SSH MODEL}

The Gonome DPC under consideration is sketched in Fig. 3(a). It is a 2D array of dielectric cylinders $\varepsilon$ arranged on a square lattice obeying $C_{4 v}$ symmetry. The lattice constant is $a_{0}$. Each unit cell now consists of one bigger cylinder of radius $R_{0}$ at the center surrounded by four smaller cylinders of radius $r_{0}=0.55 R_{0}$ at the corners. The symmetries of this system guarantee that Berry curvature vanishes everywhere in the first BZ as before [22].

Topological transitions can be induced in this DPC by simply tuning the ratio $\kappa=R_{0} / a_{0}$. To exemplify this, we plot in Figs. 3(b) and 3(c) the band structure for $\kappa=1 / 7$ and $1 / 4$, respectively. In total seven bands are shown in each case. The spatial profiles of the EM waves for each "optical" band, numbered 1-6 in Fig. 3(b), with finite frequency at $\Gamma$, which is the high-symmetry point of the first BZ, are displayed in Fig. 6(d), according to the character table of the $C_{4 v}$ group. The acoustic band lying the lowest in frequency is not sensitive to $\kappa$ and does not contribute to Zak's phase. As expected, for small $\kappa$ the intracell coupling dominates the band structure, and Zak's phase vanishes, yielding a topologically trivial band structure. Increasing $\kappa$ gives rise to several band inversions (see Appendix B for details). Beyond a critical value, which is numerically found to be $\sim 0.23$, the system ends up with the three topologically nontrivial band gaps, each having the (a)

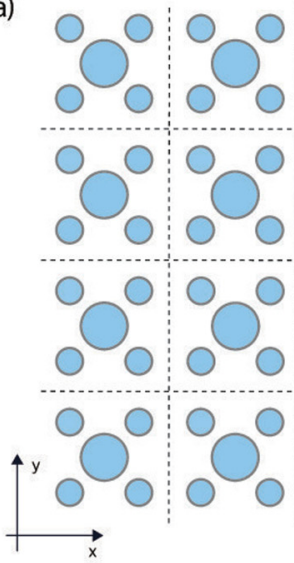

(b)

(b)

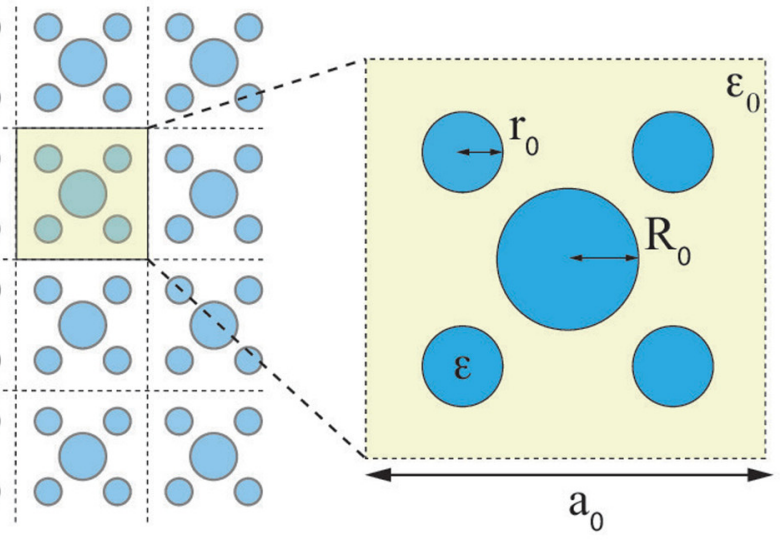

(d)
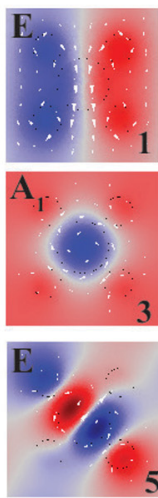
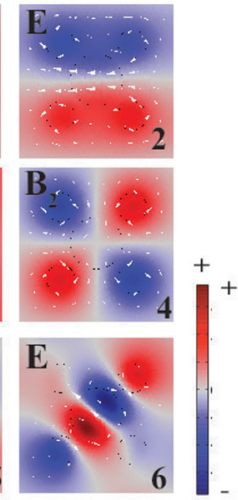

Trivial case: $\mathrm{a}_{0}=7 \mathrm{R}_{0}$
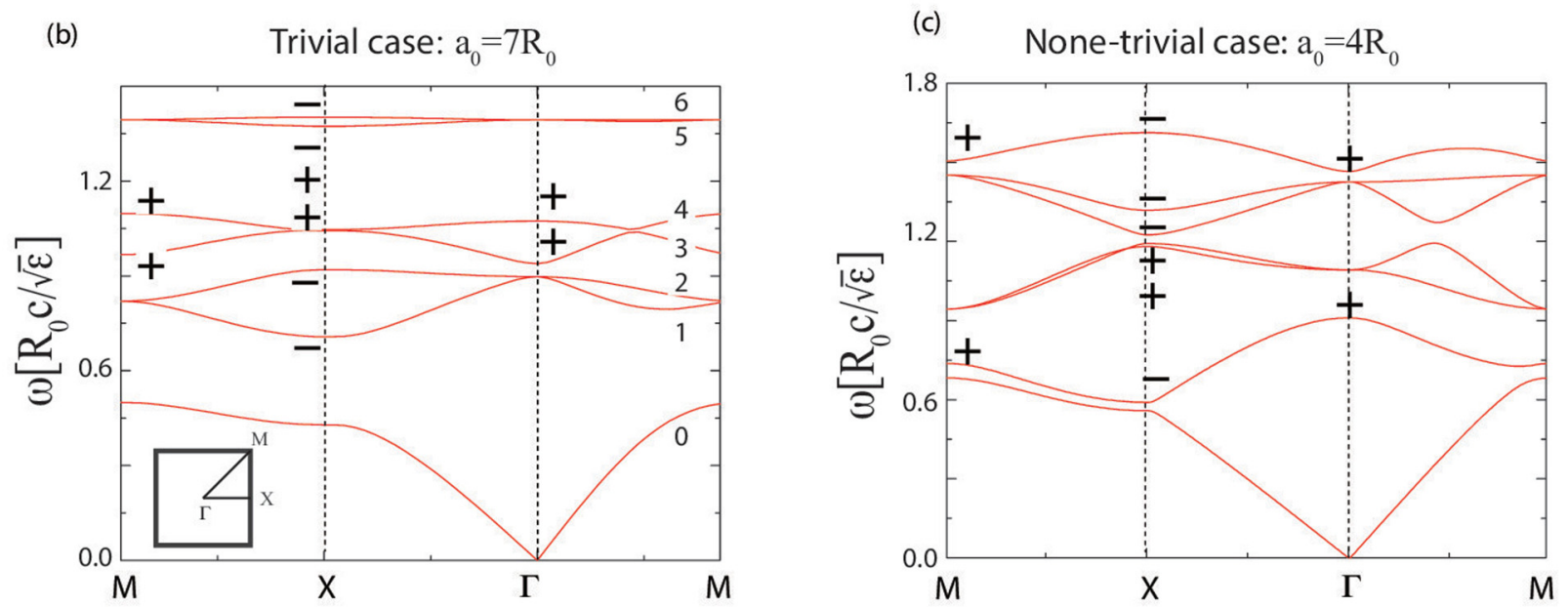

FIG. 3. (a) Schematic of the Gonome DPC. Each unit cell consists of a large cylinder of radius $R_{0}$ placed at the center of the cell and one small cylinder of radius $r_{0}$ at each of the four corners. All cylinders are made of the same dielectrics $\varepsilon$. Topological transitions are induced by tuning $\kappa=R_{0} / a_{0}$. (b) and (c) Band structures for the topologically trivial case with $a_{0}=7 R_{0}$ and the nontrivial case with $a_{0}=4 R_{0}$, respectively. Parities at high-symmetry points are indicated by plus and minus symbols. (d) Spatial profiles of the six EM modes at point $\Gamma$. Their symmetries are indicated according to the character table of $C_{4 v}$ PGS. 
(a)

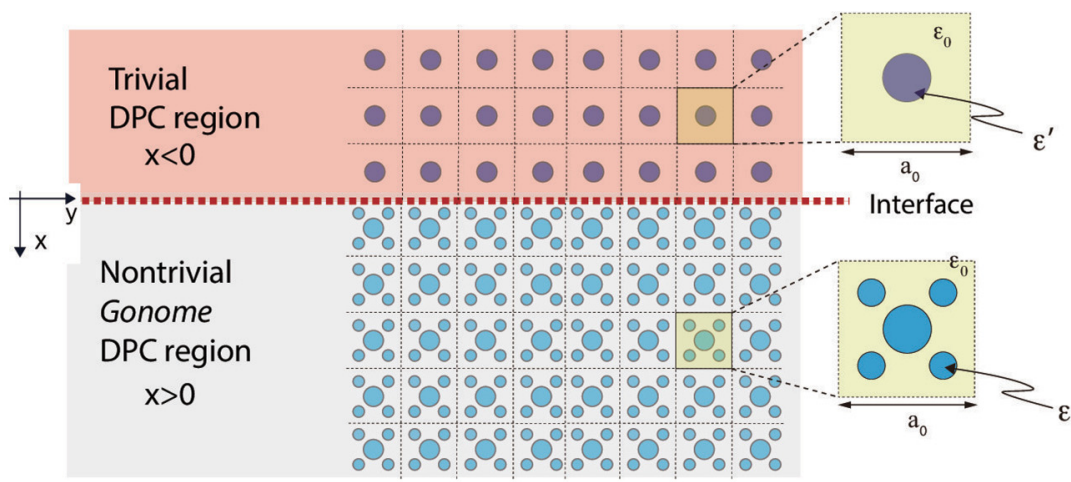

(c)

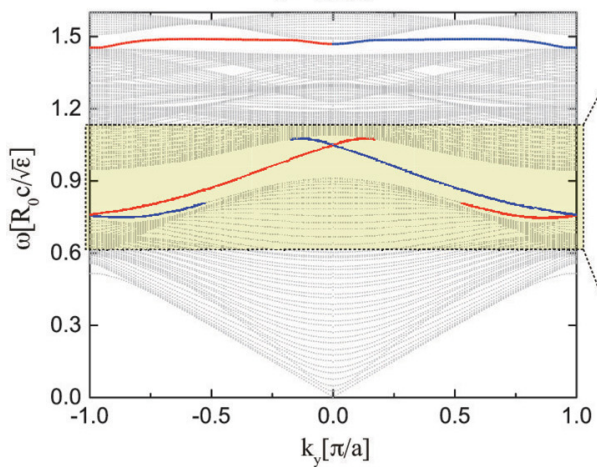

(b)
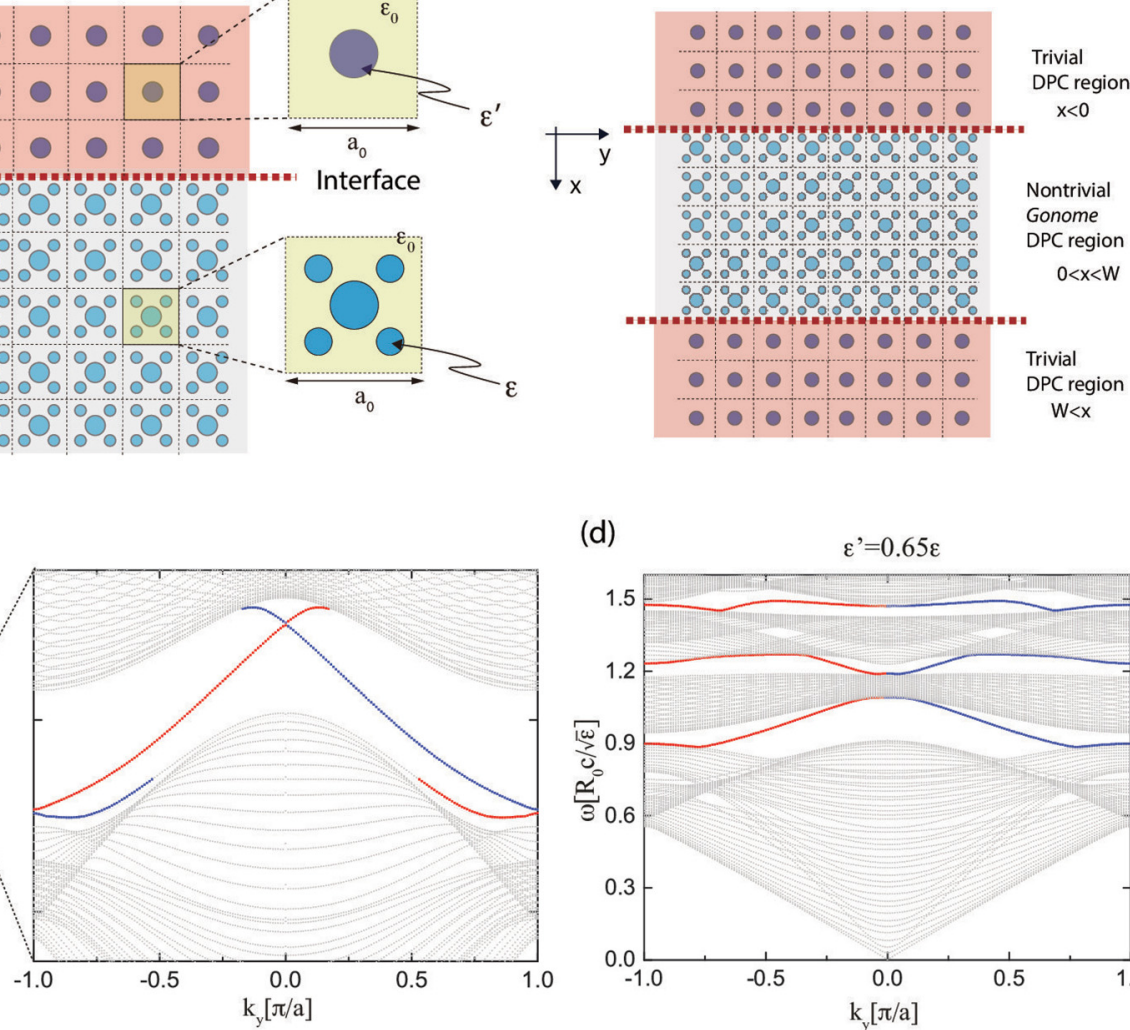

(d)

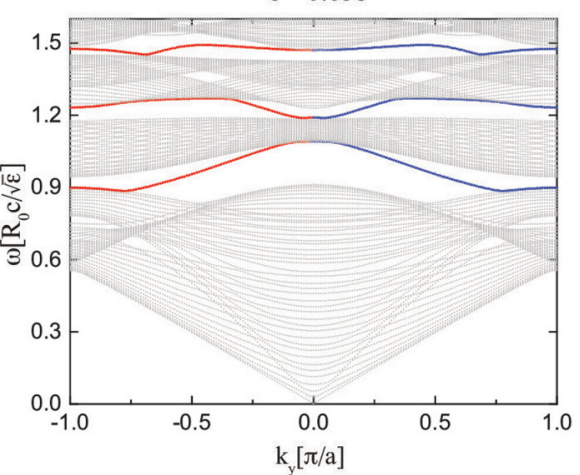

FIG. 4. (a) Interface between the Gonome DPC and a topologically trivial DPC. The dielectric constant of the cylinders is $\epsilon$ in the Gonome DPC but $\epsilon^{\prime}$ in the trivial DPC. (b) Ribbon structure: a strip of a nontrivial Gonome DPC is sandwiched in between two trivial DPCs. (c) and (d) Band structures for the ribbon with $\epsilon^{\prime}=1.15 \epsilon$ and $\epsilon^{\prime}=0.65 \epsilon$, respectively. In each band gap there appears a pair of doubly degenerate TEM bands, and the time-reversal partners are indicated in red and blue. The TEM dispersion depends on $\varepsilon^{\prime}$. In (c), where $\epsilon^{\prime}=1.15 \epsilon$, the dispersion resembles a quadratic Dirac cone at $k_{y}=0$ and $k_{y}=\pi$ (see the zoom), whereas in (d) with $\epsilon^{\prime}=0.65 \epsilon$, it is almost flat near those points.

vectored Zak's phase amounting to $(\pi, \pi)$, as inferred from the parities shown in Fig. 3(c). The magnitude of these band gaps is $\sim 6.0 \mathrm{GHz}$ for $R_{0}=0.145 \mathrm{~mm}$ and $\varepsilon=11.68$.

The Gonome DPC differs from the DPCs discussed in the preceding section in an important way. Unlike the 1D and 2D SSH models, the topologically trivial and nontrivial Gonome DPCs cannot coincide in real space by a simple translation and thus possess drastically different band structures. This suggests that there is no critical point for the topological phase transition, unlike the cases in the 1D and 2D SSH models.

We proceed to study the TEMs that are bound to appear on the interface between the topologically nontrivial Gonome DPC and a topologically trivial DPC. The interface is shown in Fig. 4(a). We have considered two types of trivial DPCs in this context. The one shown in Fig. 4(a) consists of a square array of dielectric cylinders of dielectric constant $\varepsilon^{\prime}$ (see Appendix C for details). In Appendix D, results are presented with the trivial photonic crystal replaced by a perfect electric conductor (PEC). In Fig. 4(b), a ribbon structure is formed in the region of $0<x<W$. The band structures are computed by directly solving Maxwell's equations using the FEM method. The supercell used for this computation obeys the Floquet periodic boundary condition along the $y$ direction with the phase shift determined by the wave number $k_{y}$. The resulting photonic band structures for the ribbon are shown in Figs. 4(c) and 4(d), where we see that TEMs (blue and red curves) appear inside the topological photonic band gaps, in addition to the bulk states (gray curves). Inside one of the gaps, a pair of TEM bands emerge, and Dirac cones form at $k=0$ and $k=\pi$, as shown in the close-up of Fig. 4(c), which is similar to the case of conventional topological insulators [8]. The blue and red curves represent TEMs that are time-reversal partners of each other. The TEM bands are doubly degenerate in the entire BZ, and the TEMs with opposite parities are localized at different interfaces. The TEM dispersion depends on $\varepsilon^{\prime}$. For example, for $\epsilon^{\prime}=1.15 \epsilon$ shown in Fig. 4(c), the dispersion resembles a Dirac cone at $k_{y}=0$ and $k_{y}=\pi$, whereas for $\epsilon^{\prime}=0.65 \epsilon$ in Fig. 4(d), it becomes almost flat near these points.

Finally, we discuss the robustness of TEMs. As mentioned in Sec. II, these TEMs are the consequence of $1 / 2$ charge (macroscopic) polarization due to inversion symmetry. As long as the alternation of parities at inversion-invariant points of $\mathrm{BZ}$ remains, perturbations will have no significant effect on these TEMs. To demonstrate the robustness of TEMs, we consider two types of perturbations, as shown in Figs. 5(a) and 5(b). In Fig. 5(a), we add random values up to $0.2 \varepsilon$ to the dielectric constant of randomly selected big cylinders in the nontrivial region. In Fig. 5(b), we tear up the sample, which results in curved edges. For these two finite samples, we solve the eigenvalue problem. As displayed by Figs. 5(a) 
(a)

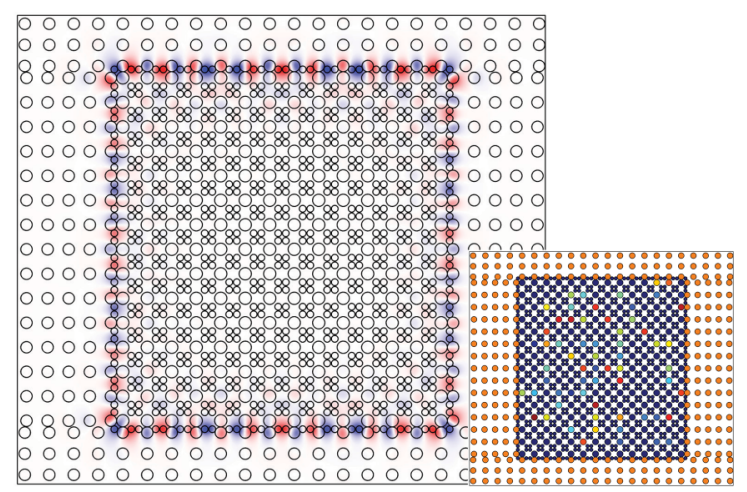

(b)

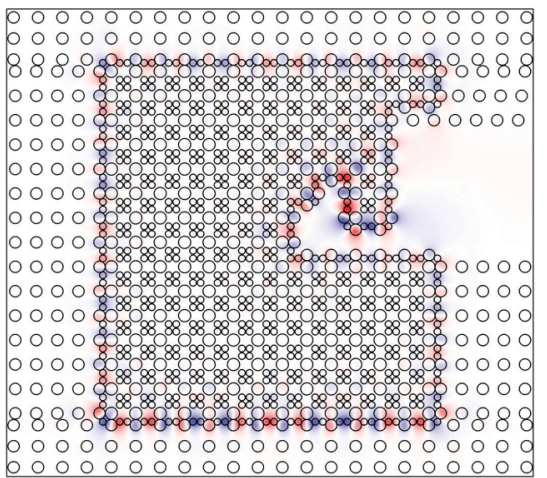

FIG. 5. TEMs (the electric field along the out-of-plane direction) in the defected samples, where blue and red represent positive and negative values of electric field. In (a), we add random values up to $0.2 \varepsilon$ to the dielectric constant of randomly selected bigger cylinders in the nontrivial region. The inset shows those defected cylinders in different colors compared with normal ones. In (b), we tear up the sample, which results in highly curved edges. In both cases, the TEMs exist with the same eigenfrequency as those in the unperturbed sample.

and 5(b), TEMs (electric field along the out-of-plane direction) exist in both defected samples. Furthermore, these edge states have the same eigenfrequency as those in the unperturbed sample.

Due to the topological protection, these TEMs are good candidates for transferring EM waves [52]. However, due to the lack of chirality associated with (pseudo)spins, finite backscattering could happen depending on the strengths of the disorders. We show that these TEMs preserve high efficiency in the presence of weak lattice disorder and imperfections by solving harmonic propagation problems. Four types of disorders are investigated for this purpose (see Fig. 6). In each case, a source, marked by a green star, emanates a harmonic wave and passes through the interface [52]. The transmission probability and the time-averaged energy flow are computed using the commercial software COMSOL. Figure 6(a) displays the transmission when a lattice mismatch is present at the edge. Near the TEM frequency the transmission can reach almost $100 \%$ in spite of the lattice mismatch. Figure 6(c) shows the energy flow passing around a metallic obstruction near the edge. In comparison with the lattice mismatch, a larger backscattering takes place, with the maximum transmission being around $75 \%$ for the case of metallic obstruction. In Figs. 6(d) and 6(e), situations are shown with a broken unit and turning around a sharp orthogonal corner, with maximum transmissions being around $100 \%$ and $80 \%$, respectively.

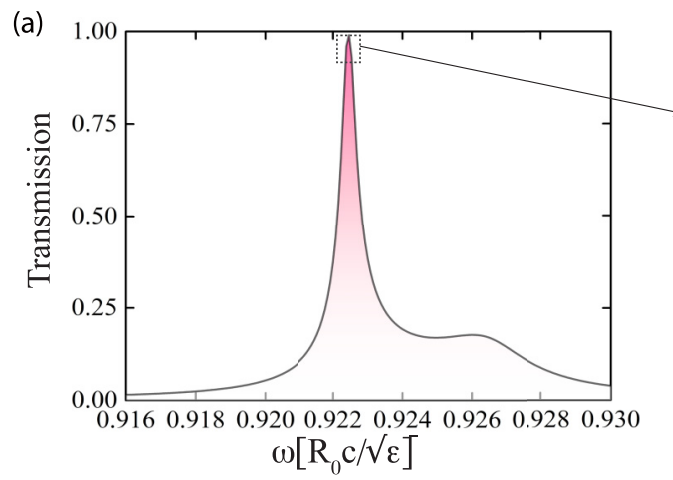

(b)

lattice mismatch

(c) metallic obstruction

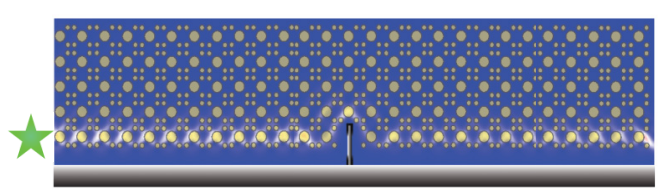

PEC

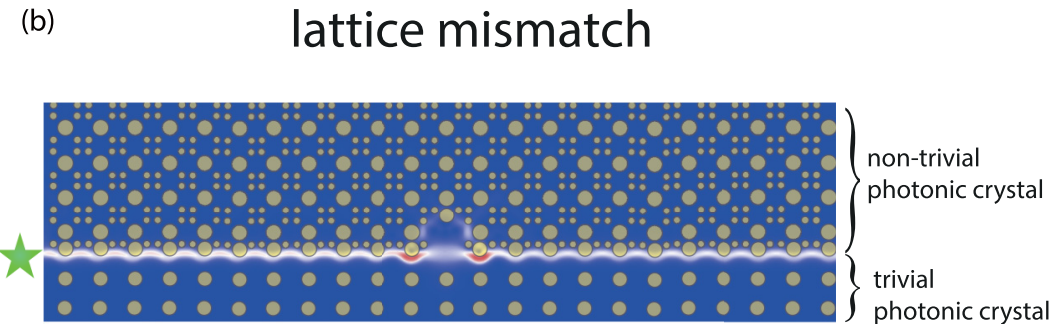

(e) 90 degrees corner

(d) broken unit

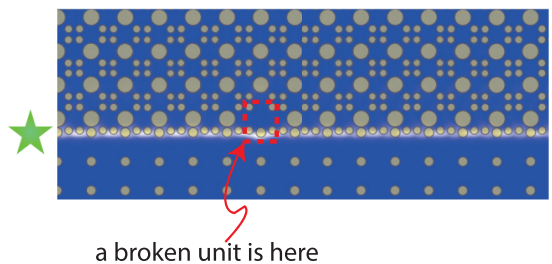

a broken unit is here

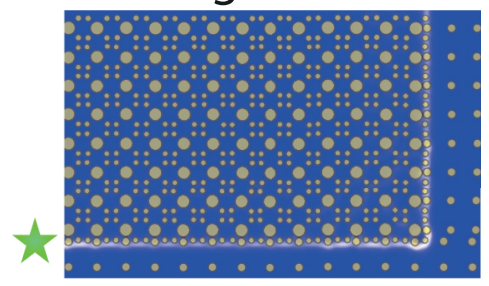

FIG. 6. Demonstration of the robustness of the TEMs against various types of disorders: (a) lattice mismatch, (b) metallic obstruction, (c) broken unit, and (d) $90^{\circ}$ corner. A harmonic EM wave is generated by a source, marked by a green star, and partially transmitted through the interface. Transmission probability and energy flow (indicated in white) are computed using the commercial software COMSOL. The transmission for the lattice mismatch is displayed in (a), where we see that the peak transmission reaches around 100\%. Comparably high transmission has been observed for the obstructions shown in (b), (c), and (d). 


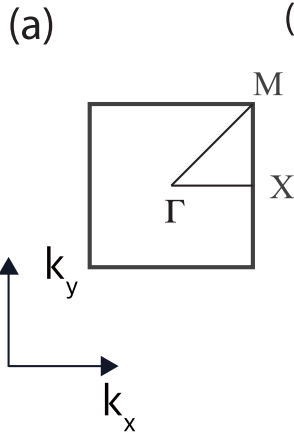

(b)

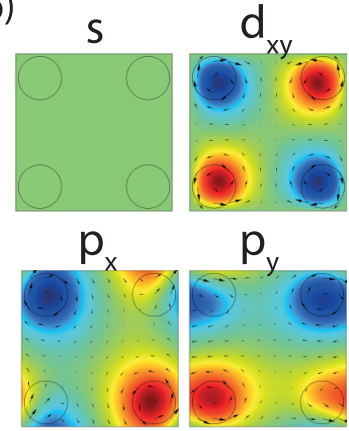

(c)

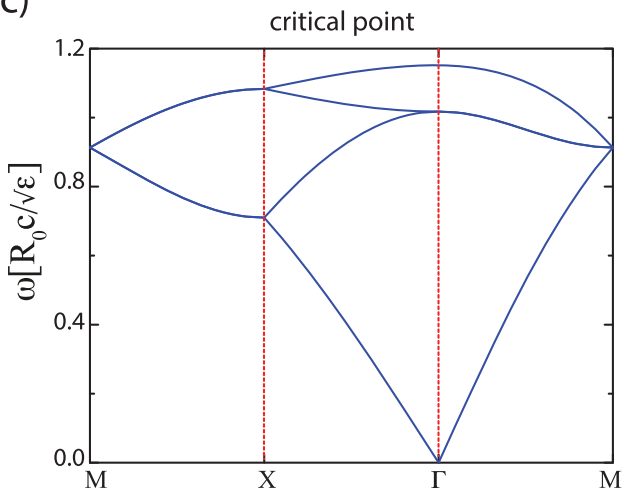

(e)

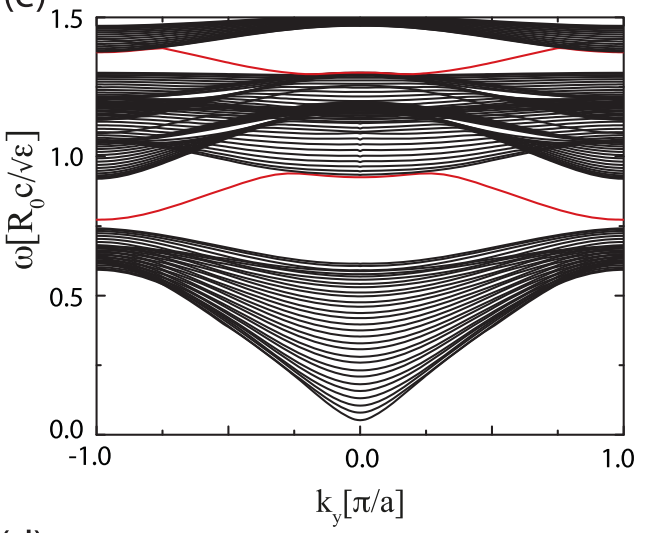

(d)

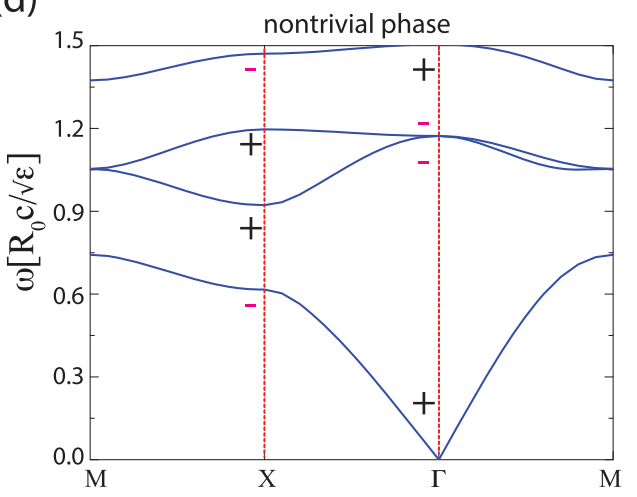

FIG. 7. (a) The corresponding first BZ of the Shinome DPC. (b) Spatial distributions of four EM modes at point $\Gamma$ involved in the band engineering, which are isolated $s$ and $d_{x y}$ modes and degenerate $p$ modes. The isolated modes have even parity, while the degenerate ones have odd parity. Colors indicate the electric field in the out-of-plane direction, and arrows present the magnetic field of in-plane directions. (c) Energy spectrum of the bulk for the Shinome DPC with the critical lattice constant $a_{0}=10 r$, where the energy bands of four EM modes touch at high-symmetric points of the first BZ. (d) Energy spectrum of the bulk for the Shinome DPC with $a_{0}<10 r$, where two topological photonic band gaps open. The parities of each band at high-symmetry points are denoted by the plus and minus signs. Since the energy bands below the gap at the points $X(Y)$ and $\Gamma$ have opposite parities, the two photonic band gaps are topologically nontrivially protecting the vectored Zak's phase $(\pi, \pi)$. (e) Energy band structure for the ribbon of the Shinome DPC with the periodic boundary condition along the interface direction. The ribbon is made of the Shinome DPC with $a_{0}<10 r$ bounded by a trivial photonic crystal. TEMs marked by red curves appear in the two photonic band gaps beside the bulk states marked by black curves.

These results demonstrate that the TEMs of the Gonome DPC are robust against various defects and may be used as efficient photonic waveguides. In principle, the robustness of the TEMs is determined by the difference between the intracell and intercell couplings [23].

\section{SUMMARY}

In summary, we have studied photonic crystals with easyto-fabricate structures that mimic either the 2D SSH model or its extension. All of them can possess topologically nontrivial band gaps and, upon being terminated, robust edge states. The topological properties are characterized by the vectored Zak's phase rather than the conventional topological invariants such as the Chern number on the basis of nonvanishing Berry curvature. In the systems studied here, Berry curvature vanishes. Our results add a new perspective to the current understanding of topological emergence, and the structures we have designed may be used as efficient wave guides. We believe this work will stimulate further experimental and theoretical interest in this topic.

\section{ACKNOWLEDGMENTS}

We thank R. Yu, T. Kawakami, L.-H. Wu, X. Hu, F. Nori, $\mathrm{Zh}$. Li, Y. Xu, and M. Lein for useful discussions. F.L. is an overseas researcher under the Postdoctoral Fellowship of Japan Society for the Promotion of Science (JSPS). This work was supported by JSPS KAKENHI Grants No. JP25107005, No. JP15K21722, No. JP15K13507, and No. JP17F17326.

\section{APPENDIX A: PHOTONIC STATES OF THE SHINOME DPC}

In this appendix, we discuss a simple and straightforward realization of Eq. (1) in the main text by the Shinome DPC, whose schematic structure is shown in Fig. 2(b). We name this crystal structure the "Shinome" crystal because each unit cell of this DPC contains four identical cylinders resembling the quatre face of a die (similarly, the Gonome structure resembles the cinque face of a die). The radius of the four cylinders is $r_{0}$, the dielectric constant is $\varepsilon$, and they are located at $\left( \pm 2.5 r_{0}, \pm 2.5 r_{0}\right)$. The lattice constant is $a_{0}$. Figure 7(a) shows the corresponding first BZ. Figure 7(b) display the spatial 
distribution of the four EM modes used in the band engineering, which are $s, d_{x y}$, and a pair of $p$ EM modes. From Fig. 7(b) we see that the degenerate $p$ modes have an odd parity, while the isolated $s$ and $d_{x y}$ modes have an even parity.

As $a_{0}$ decreases, the distance between the cells becomes smaller, and thus, $\left|\gamma^{\prime} / \gamma\right|$ increases effectively. It should be noted that all the cylinders are placed with equal distance for the whole system when $a_{0}=10 r_{0}$, i.e., $\gamma^{\prime}=\gamma$. In this condition, the Shinome DPC is at the critical point of the topological phase transition characterized by the vectored Zak's phase. The energy spectrum at the critical point of the Shinome DPC is shown in Fig. 7(c), where four energy bands touch at the highsymmetry points of the first BZ. As $a_{0}$ decreases further, three topological photonic band gaps open simultaneously, which are characterized by Zak's phase $(\pi, \pi)$ owing to the opposite parities at $X(Y)$ and $\Gamma$ points, as shown in Fig. 7(d), where the parities are denoted by the plus and minus signs.

The nonzero Zak's phase manifests as a TEM once if we make the surface or edge by considering the ribbon-shaped boundary condition. To confirm this, we consider an interface between the topological Shinome DPC and the trivial DPC that possesses wider photonic band gaps covering that of the Shinome DPC completely. The energy spectrum for such a supercell with Floquet periodic boundary conditions along the interface direction is shown in Fig. 7(e). From Fig. 7(e) we observe that the edge states (red curves) appear in the topological photonic band gaps beside the bulk states (black curves).

Here we mention the actual parameters used in the numerical simulations for the sake of the experimental realization of the Shinome DPC. The radius of four cylinders is chosen to be $0.08 \mathrm{~mm}$, and the dielectric constant is 100 . One can set the dielectric constant to any desired material such as silicon. The lattice constant for the nontrivial phase is $0.58 \mathrm{~mm}$, and the typical frequency of such a photonic crystal is $65 \mathrm{GHz}$. The structure of the trivial photonic crystal used for constructing the interface is simply one cylinder centered at the unit cell, with a radius of $0.135 \mathrm{~mm}$ and a dielectric constant of 115 . It is noted that all the parameters are scalable in Maxwell's equations. Although we focused on DPCs here, metallic photonic crystals are also expected to show similar results as long as the intercellular hopping is larger than the intracellular one. (a)
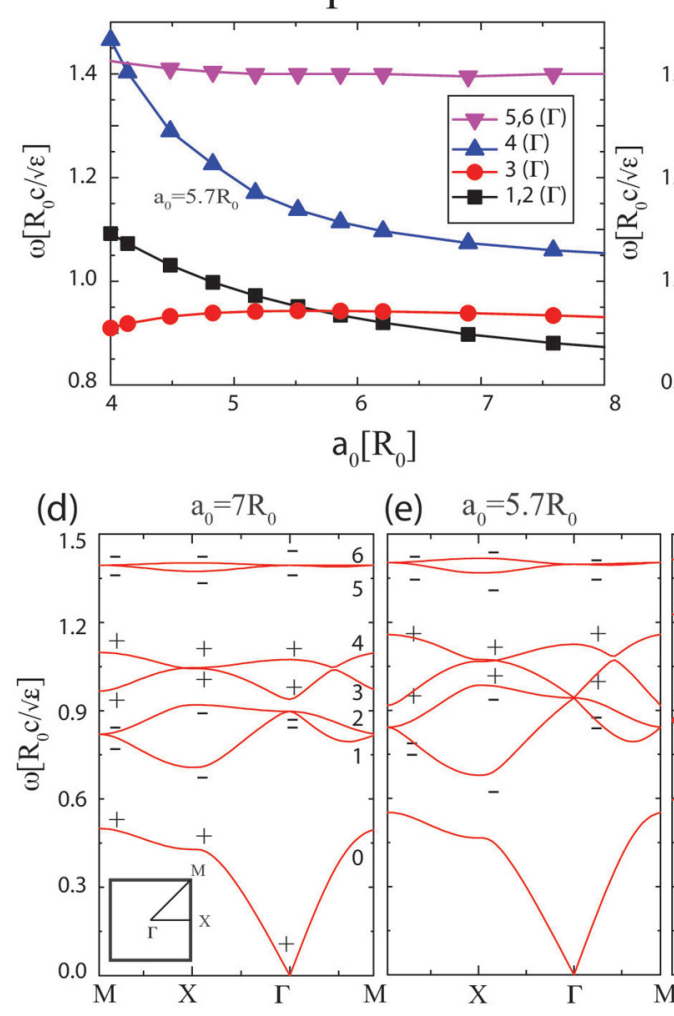

(b)

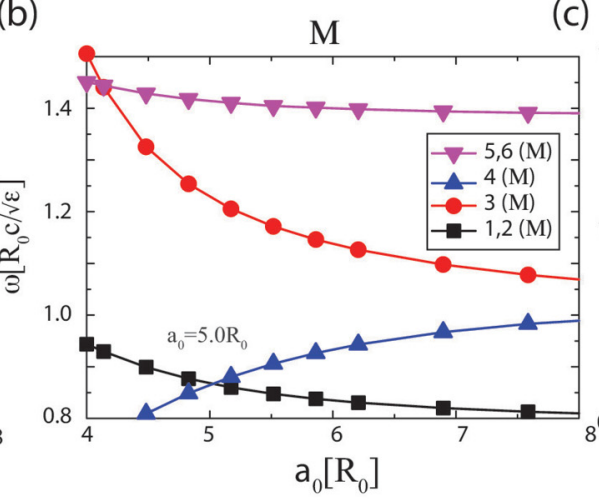

(f) $\mathrm{a}_{0}=5.0 \mathrm{R}_{0}$

(g) $\mathrm{a}_{0}=4.5 \mathrm{R}_{0}$

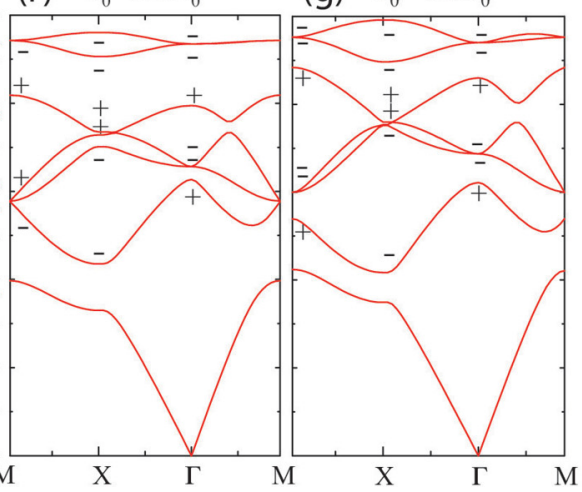

(c)

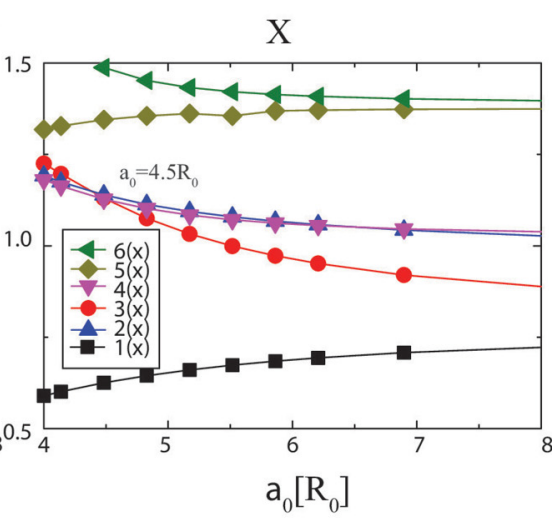

(h)

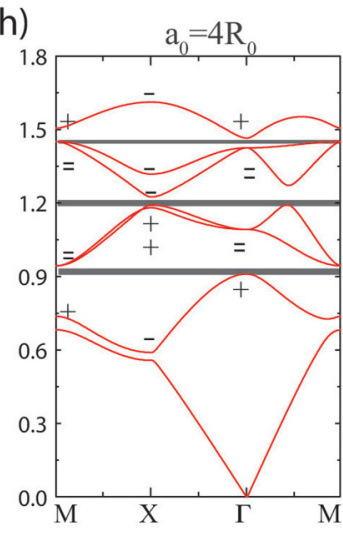

FIG. 8. The lattice constant dependence of eigenfrequencies for the six lowest EM modes at high-symmetry points: (a) $\Gamma$, (b), $M$ and (c) $X$. Photonic energy band structures for the lattice constant of (d) $a_{0}=7 R_{0}$, (e) $5.7 R_{0}$, (f) $5.0 R_{0}$, and (g) $4.5 R_{0}$. These bands can be sorted out according to their spatial transformation properties under $C_{4 v}$ symmetric operations. In the specified range of $a$, the lowest zeroth band always transforms like $z$ with $q_{0}=0$. The upper six bands make nondegenerated $A_{1}$ - and $B_{2}$-type and degenerated $E$-type irreducible representations of the $C_{4 v}$ group. The $A_{1}$ - and $B_{2}$-type bands transform like $x^{2}+y^{2}$ and $x y$, while the $E$-type ones transform like $(x, y)$. The parities of each band are marked by the plus and minus signs. For $a>5.7 R_{0}$, all bands have $q_{n}=0$, and therefore, the structure is topologically trivial with a vanishing Zak's phase. When $a_{0}$ decreases, band inversions take place, and some of the bands acquire $q_{n} \neq 0$, as plotted in (e) to (g). (h) Finally, at $a_{0}=4.0 R_{0}$, the band structure features three band gaps marked by gray bars, each being topologically nontrivial with nonvanishing Zak's phases $(\pi, \pi)$. 


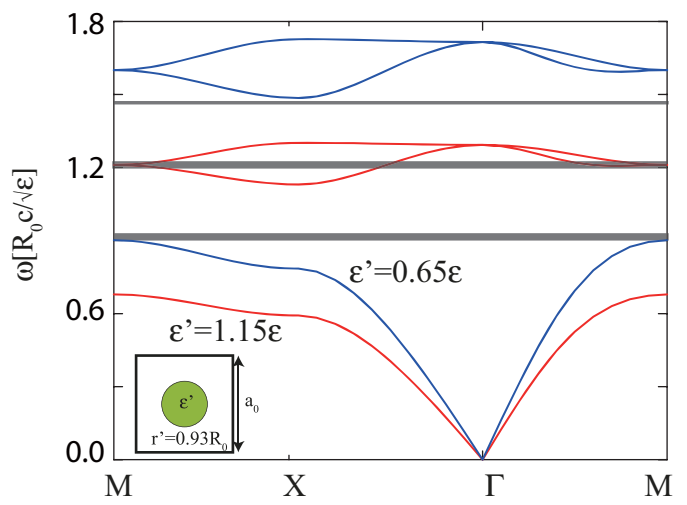

FIG. 9. Energy band structure for the trivial photonic crystal used for forming boundaries. The inset displays the unit cell of the trivial photonic crystal, which is composed of a single dielectric cylinder $\epsilon^{\prime}$ and respects $C_{4 v}$ symmetry. Two $\epsilon^{\prime}$ used in the main text are shown by red and blue curves for $\epsilon^{\prime}=1.15 \epsilon$ and $\epsilon^{\prime}=0.65 \epsilon$, respectively. The three topological gaps of the proposed crystal are marked by gray bars.

\section{APPENDIX B: EVOLUTION OF ENERGY BANDS BY TUNING $a_{0}$}

The details of the evolution of photonic band structures in the Gonome DPC by decreasing $a_{0}$ are summarized here. Figures 8(a) to 8(c) show the lattice constant dependence of the eigenfrequency for the lowest six EM modes at high-symmetry points $M, X$, and $\Gamma$ points, respectively. As seen from Figs. 8(a) and 8(b), the $d_{x y}$-orbital-like EM modes (energy band 4) have opposite trends at $\Gamma$ and $M$ points when the lattice constant decreases, which makes the band inversion among $p$-orbitallike (energy bands 1 and 2) and $d$-orbital-like (energy bands 3 and 4) EM modes possible.

Figures 8(d) to 8(h) display the energy spectra of the Gonome DPC for the bulk at several $a_{0}$ values varying from $a_{0}=7 R_{0}$ to $a_{0}=4 R_{0}$. As seen from Figs. 8(d) to 8(h), after three band inversions, three topological photonic band gaps with nonzero Zak's phases form.

\section{APPENDIX C: DESIGN OF THE TRIVIAL PHOTONIC CRYSTAL USED FOR BOUNDARIES}

Different from electronic systems, the interface for photonic systems cannot be built between the photonic crystal and vacuum because, generally, the EM waves leak into the free space. To confine the EM field, one can use a trivial photonic crystal to terminate the nontrivial one. The unit cell of the trivial DPC used for the termination of the topological Gonome DPC in the main text is shown in the inset of Fig. 9. This trivial DPC is composed of a single dielectric cylinder with a radius $r^{\prime}=0.93 R_{0}$ arranged in a square lattice with the same lattice constant as the topological Gonome DPC. The trivial DPC is designed to preserve the $C_{4 v}$ point-group symmetry. The bulk spectra for the DPC with dielectric constants $\epsilon^{\prime}=1.15 \epsilon$ and $\epsilon^{\prime}=0.65 \epsilon$ are shown in Fig. 9 by red and blue curves, respectively. For the case with $\epsilon^{\prime}=1.15 \epsilon$, the energy band structure for the bulk of the trivial DPC covers the second topological photonic band gap, which is indicated by gray bars. (a)

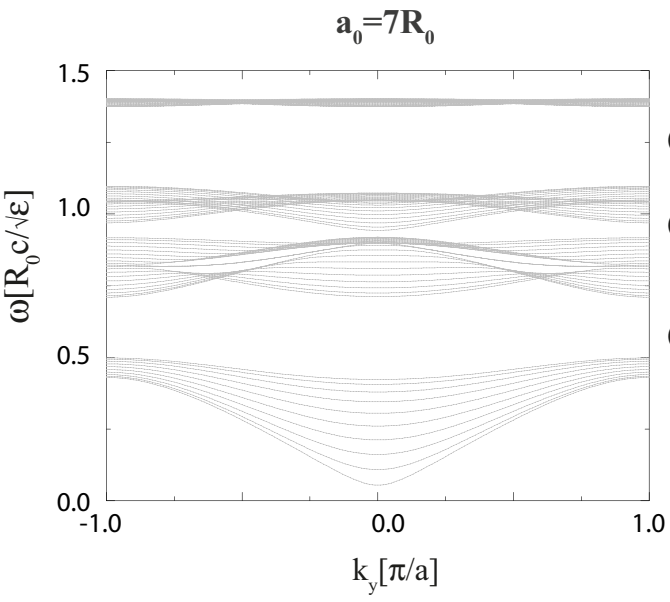

(c)

1st gap (b)

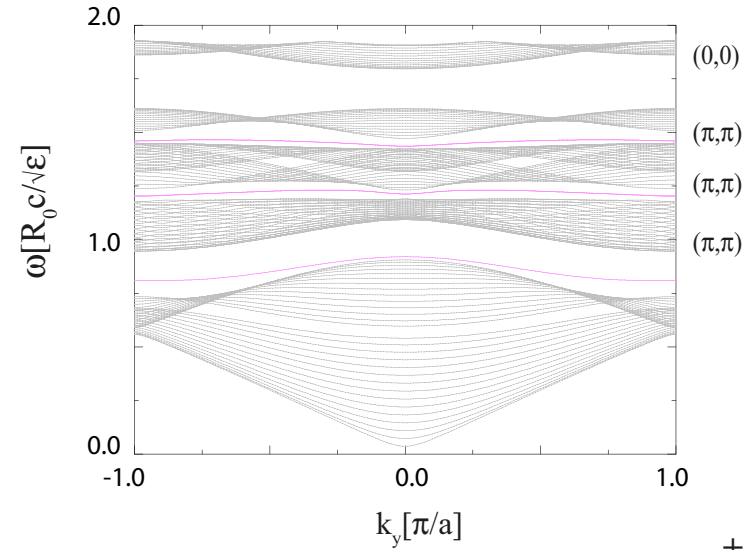

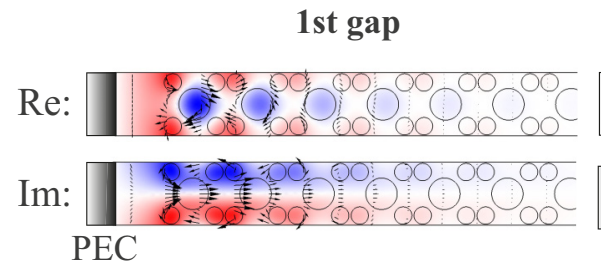
$(0,0)$ $(0,0)$ $(0,0)$

2nd gap 3rd gap
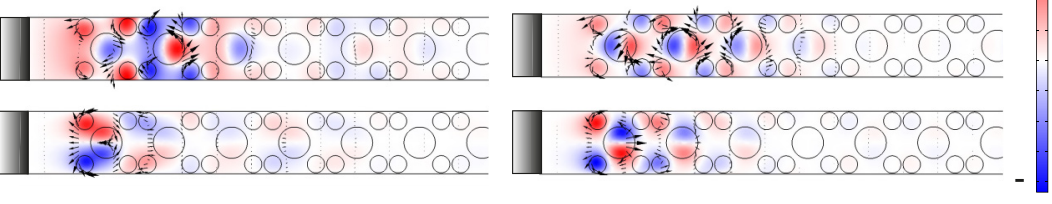

FIG. 10. (a) and (b) The ribbon spectra of proposed photonic crystals terminated by a perfect electric conductor with $a=7.0 R_{0}$ and $a_{0}=4 R_{0}$, respectively. There are no edge modes in the structure with $a_{0}=7.0 R_{0}$, where all gaps are trivial with vanishing Zak's phases. There are three nontrivial gaps in the structure with $a_{0}=4 R_{0}$, with each gap hosting a band of edge modes (pink curves). Note that the edge modes are not continuously connected to the bulk bands because $C_{4 v}$ symmetry is broken on the edge. (c) Spatial profiles of the edge modes, where the complex electric field (indicated by color) and the magnetic field (arrows) are illustrated for the modes at $k_{y}=-0.3$ in a unit cell. 


\section{APPENDIX D: TOPOLOGICAL EDGE STATES FOR PERFECT ELECTRIC CONDUCTOR BOUNDARIES}

Besides the trivial photonic crystals, PECs can also be used as the confining boundary of the nontrivial DPC. We display the energy spectra for the ribbons of the Gonome DPC terminated by the PEC in Figs. 10(a) and 10(b) for large and small $a_{0}$, respectively. In the trivial case of $a_{0}=7 R_{0}$, there is no edge state in the gaps, while for the nontrivial case of $a_{0}=4 R_{0}$, three edge states appear. However, as $C_{4 v}$ symmetry is broken near the edges by the PEC boundary condition, the dispersion of the edge states does not bridge the bulk ones. The spatial profiles of these three edge states are shown in Fig. 10(c); one sees that the edge states are indeed localized near the edges.
[1] D. J. Thouless, Topological Quantum Numbers in Nonrelativistic Physics (World Scientific, Singapore, 1998).

[2] M. Z. Hasan and C. L. Kane, Rev. Mod. Phys. 82, 3045 (2010).

[3] X.-L. Qi and S.-C. Zhang, Rev. Mod. Phys. 83, 1057 (2011).

[4] L. Lu, J. D. Joannopoulos, and M. Soljačić, Nat. Photonics 8, 821 (2014).

[5] A. Bansil, H. Lin, and T. Das, Rev. Mod. Phys. 88, 021004 (2016).

[6] K. v. Klitzing, G. Dorda, and M. Pepper, Phys. Rev. Lett. 45, 494 (1980).

[7] C. L. Kane and E. J. Mele, Phys. Rev. Lett. 95, 226801 (2005).

[8] B. A. Bernevig, T. L. Hughes, and S.-C. Zhang, Science 314, 1757 (2006).

[9] L. Fu, C. L. Kane, and E. J. Mele, Phys. Rev. Lett. 98, 106803 (2007).

[10] J. E. Moore and L. Balents, Phys. Rev. B 75, 121306 (2007).

[11] M. König, S. Wiedmann, C. Brüne, A. Roth, H. Buhmann, L. W. Molenkamp, X.-L. Qi, and S.-C. Zhang, Science 318, 766 (2007).

[12] D. Hsieh, D. Qian, L. Wray, Y. Xia, Y. S. Hor, R. J. Cava, and M. Z. Hasan, Nature (London) 452, 970 (2008).

[13] R. Roy, Phys. Rev. B 79, 195321 (2009).

[14] L. Fu, Phys. Rev. Lett. 106, 106802 (2011).

[15] Y. Tanaka, Z. Ren, T. Sato, K. Nakayama, S. Souma, T. Takahashi, K. Segawa, and Y. Ando, Nat. Phys. 8, 800 (2012).

[16] P. Dziawa, B. J. Kowalski, K. Dybko, R. Buczko, A. Szczerbakow, M. Szot, E. Łusakowska, T. Balasubramanian, B. M. Wojek, M. H. Berntsen, O. Tjernberg, and T. Story, Nat. Mater. 11, 1023 (2012).

[17] X. Wan, A. M. Turner, A. Vishwanath, and S. Y. Savrasov, Phys. Rev. B 83, 205101 (2011).

[18] S. Borisenko, Q. Gibson, D. Evtushinsky, V. Zabolotnyy, B. Büchner, and R. J. Cava, Phys. Rev. Lett. 113, 027603 (2014).

[19] R. Yu, Q. Wu, Z. Fang, and H. Weng, Phys. Rev. Lett. 119, 036401 (2017).

[20] D. J. Thouless, M. Kohmoto, M. P. Nightingale, and M. den Nijs, Phys. Rev. Lett. 49, 405 (1982).

[21] F. D. M. Haldane, Phys. Rev. Lett. 61, 2015 (1988).

[22] D. Xiao, M.-C. Chang, and Q. Niu, Rev. Mod. Phys. 82, 1959 (2010).

[23] F. Liu and K. Wakabayashi, Phys. Rev. Lett. 118, 076803 (2017).

[24] F. D. M. Haldane and S. Raghu, Phys. Rev. Lett. 100, 013904 (2008).

[25] Z. Wang, Y. D. Chong, J. D. Joannopoulos, and M. Soljacić, Phys. Rev. Lett. 100, 013905 (2008).

[26] Z. Wang, Y. Chong, J. D. Joannopoulos, and M. Soljacić, Nature (London) 461, 772 (2009).
[27] C. He, X.-L. Chen, M.-H. Lu, X.-F. Li, W.-W. Wan, X.-S. Qian, R.-C. Yin, and Y.-F. Chen, J. Appl. Phys. 107, 123117 (2010).

[28] M. Hafezi, E. A. Demler, M. D. Lukin, and J. M. Taylor, Nat. Phys. 7, 907 (2011).

[29] K. Fang, Z. Yu, and S. Fan, Nat. Photonics 6, 782 (2012).

[30] M. C. Rechtsman, J. M. Zeuner, Y. Plotnik, Y. Lumer, D. Podolsky, F. Dreisow, S. Nolte, M. Segev, and A. Szameit, Nature (London) 496, 196 (2013).

[31] H. Cheng, L. Lin, X.-C. Sun, X.-P. Liu, M.-H. Lu, and Y.-F. Chen, Int. J. Mod. Phys. B 28, 1441001 (2014).

[32] M. Hafezi, S. Mittal, J. Fan, A. Migdall, and J. M. Taylor, Nat. Photonics 7, 1001 (2013).

[33] A. B. Khanikaev, S. H. Mousavi, W.-K. Tse, M. Kargarian, A. H. MacDonald, and G. Shvets, Nat. Mater. 12, 233 (2013).

[34] F. Liu and J. Li, Phys. Rev. Lett. 114, 103902 (2015).

[35] K. Y. Bliokh, D. Smirnova, and F. Nori, Science 348, 1448 (2015).

[36] H.-Y. Deng, F. Liu, and K. Wakabayashi, Europhys. Lett. 114, 35002 (2016).

[37] L.-H. Wu and X. Hu, Phys. Rev. Lett. 114, 223901 (2015).

[38] L. Xu, H.-X. Wang, Y.-D. Xu, H.-Y. Chen, and J.-H. Jiang, Opt. Express 24, 18059 (2016).

[39] L. Lu, Z. Wang, D. Ye, L. Ran, L. Fu, J. D. Joannopoulos, and M. Soljačić, Science 349, 622 (2015).

[40] Q. Lin, M. Xiao, L. Yuan, and S. Fan, Nat. Commun. 7, 13731 (2016).

[41] C. Fang, M. J. Gilbert, and B. A. Bernevig, Phys. Rev. B 86, 115112 (2012).

[42] F. Liu and K. Wakabayashi, J. Phys. Soc. Jpn. 86, 123707 (2017).

[43] Y. Liu, C.-S. Lian, Y. Li, Y. Xu, and W. Duan, Phys. Rev. Lett. 119, 255901 (2017).

[44] J. Zak, Phys. Rev. Lett. 62, 2747 (1989).

[45] D. Vanderbilt and R. D. King-Smith, Phys. Rev. B 48, 4442 (1993).

[46] R. Resta, Rev. Mod. Phys. 66, 899 (1994).

[47] J. Zak, Phys. Rev. B 32, 2218 (1985).

[48] N. Marzari, A. A. Mostofi, J. R. Yates, I. Souza, and D. Vanderbilt, Rev. Mod. Phys. 84, 1419 (2012).

[49] K. Sakoda and H. Zhou, Opt. Express 18, 27371 (2010).

[50] In practice, the Wannier functions are often approximated by the so-called defect EM waves, which are the solutions of Eq. (3) with $\varepsilon(r)$ replaced by a DPC with a defect at unit cell $\mathbf{R}$. Such a defect can, for example, be due to a varied constituting dielectric in a unit cell.

[51] X.-D. Chen, D. Zhao, X.-S. Zhu, F.-L. Shi, H. Liu, J.-C. Lu, M. Chen, and J.-W. Dong, Phys. Rev. A 97, 013831 (2018).

[52] See Supplemental Material at http://link.aps.org/supplemental/ 10.1103/PhysRevB.97.035442 for a simulation movie of TEMs excited by a harmonic source. 